\title{
The Forward Muon Spectrometer of ALICE - Status and Commissioning results.
}

\author{
Francesco BOSSÜ* for the ALICE Collaboration \\ Università di Torino and INFN Torino \\ E-mail: bossu@to.infn.it
}

In the framework of the ALICE physics program, the goal of the muon spectrometer is the study of quarkonia and open heavy flavours production via the muonic channel in a wide range of transverse momentum and in the pseudo-rapidity range $-4.0<\eta<-2.5$.

The muon spectrometer consists of absorbers that reduce the initial flux of primary hadrons, a warm dipole and a tracking system to determine muon momenta, a muon filter (iron wall) and a trigger system that selects events with muons from quarkonia and heavy flavours decay.

The tracking system consists of ten planes of Cathode Pad Chambers (about $100 \mathrm{~m}^{2}$ in surface and about 1.1 millions of channels), while the trigger system is based on four planes of Resistive Plate Chambers (about $140 \mathrm{~m}^{2}$ in surface and $21 \mathrm{k}$ channels).

To have under control the performance of the system, an intense commissioning activity has been carried out and specific analysis tools have been developed. After a description of the muon spectrometer layout, the main results of the commissioning will be presented.

XLVIII International Winter Meeting on Nuclear Physics in Memoriam of Ileana Iori 25-29 January 2010

Bormio, Italy

\footnotetext{
* Speaker.
} 


\section{Introduction}

ALICE[1] (A Large Ion Collider Experiment) has been designed to study the properties of the medium of deconfined quarks and gluons (QGP) that is supposed to be formed at high energy density or temperature $\left(\varepsilon_{c}=0.7 \mathrm{GeVfm}^{-3}\right.$ or $\left.T_{c} \sim 180 \mathrm{MeV}\right)$ reached in ultra-relativistic heavy-ion collisions.

Hard probes, such as heavy quarkonium states, provide an essential tool to study the early and hot stage of heavy-ion collisions, as it has been clearly shown by the SPS and RHIC experiments[2, 3].

The main role of the ALICE muon spectrometer is to study the production of quarkonia and heavy quarks via their muonic decays. Theoretical models predict that the quarkonia production in a QGP can be reduced by a color screening effect or increased by statistical hadronization of uncorrelated heavy quark-antiquark pairs (see [4] and re. therein). Quarks inside a hot medium are expected to lose energy through multiple scattering with the free partons and medium induced gluon radiation, effects that can affect the spectrum of the open heavy flavour (see e.g.[5]). Furthermore, the magnitude of these effects is sensitive to the properties of the medium, like its energy density and the quark species.

In the first years of LHC operation, the ALICE physics program will be focused on $\mathrm{Pb}-\mathrm{Pb}$ and pp collisions. The latter, on top of their intrinsic interest, will provide the necessary baseline for heavy-ion data[6].

\section{Muon Spectrometer}

The design of the ALICE muon spectrometer has been essentially driven by two requirements: to perform charmonium detection down to zero transverse momentum and to resolve the bottomonium states $(\Upsilon(1 S), \Upsilon(2 S)$ and $\Upsilon(3 S))$ in the large-background environment of central $\mathrm{Pb}-\mathrm{Pb}$ collisions. The mass resolution required for the $\Upsilon$ is about $100 \mathrm{MeV} / \mathrm{c}^{2}$ at $10 \mathrm{GeV} / \mathrm{c}^{2}$.

The spectrometer, as shown in Fig.1, is composed of a system of absorbers, five stations of tracking detectors that, together with a dipole magnet, are used to measure the muon momentum and two stations of trigger chambers shielded by an iron wall. The spectrometer has a total length of $17 m$ and covers the polar angular range $172^{\circ} \leq \theta \leq 178^{\circ}(-4 \leq \eta \leq-2.5)$ with respect to the reference frame of ALICE.

\section{Absorbers}

To reduce the flux of primary hadrons, the spectrometer is shielded by a system of absorbers. A front absorber stops the flux of primary hadrons, a small angle absorber protects tracking and trigger detectors against background particles coming from secondary interactions in the beam pipe. A further protection is provided for trigger chambers by an iron wall that stops remaining hadrons. The front absorber and the muon filter stop muons with momentum less than $4 \mathrm{GeV} / \mathrm{c}$.

\section{Dipole magnet}

The magnetic field for momentum measurement is provided by one of the largest warm dipoles ever built (820tons) with a nominal magnetic field of $0.7 T$. The field integral between the interaction point and the muon filter along the beam axis is $\int|B| d z \sim 3 T m$. 




Figure 1: Layout of the muon spectrometer

\section{Tracking System}

A spatial resolution of about $100 \mu \mathrm{m}$, to achieve the required mass resolution for the $\Upsilon$, and the need of being operated in a high particle multiplicity environment are the requirements that have driven the design of the tracking chambers. These requirements are fulfilled by Cathode Pad Chambers (CPC). They are arranged in 5 stations, with two detector planes each, for a total active area of about $100 \mathrm{~m}^{2}$. Each chamber has two cathode planes, which are both read out to provide two-dimensional hit information. The first two stations are placed before the dipole magnet and have a quadrant design, while for the station 3, placed inside the magnet, and stations 4 and 5, after the magnet, a modular design was chosen[7]. The spatial resolution measured in tests with beam was found to be of the order of $50 \mu \mathrm{m}$, i.e. better than the required value[8]. Different pad densities are present in the CPCs depending on the station and on the distance from the beam pipe.

The total number of readout channels is around 1.1 million. The front-end electronics is based on a 64 channels board (MANU) including four charge amplifier chips (MANAS), ADCs and a readout chip allowing zero suppression (MARC). The data flow from each half plane is gathered by the Concentrator ReadOut Cluster (CROCUS) which sends it to the DAQ, performs calibration of the MANU and dispatches the trigger signal from the central trigger processor (CTP).

The displacements and deformations of the tracking chambers with respect to the initial geometry (due to various reasons, including switching on the magnetic field) will be measured and recorded during data taking by the Geometry Monitoring System (GMS)[9]. The GMS is an array of 460 optical sensors which are installed on platforms placed at each corner of the tracking chambers. The requirement is to monitor the position of all the tracking chambers with a resolution better than $40 \mu \mathrm{m}$.

\section{Trigger System}

Simulations indicate that, at the level of muon trigger detectors, in a central $\mathrm{Pb}-\mathrm{Pb}$ collisions up to 8 low transverse momentum $\left(p_{t}\right)$ muons from $\pi$ and $K$ decays are expected. Since muons from quarkonia and heavy flavour decays have a larger $p_{t}$, a $p_{t}$ cut is applied at the trigger level. To perform this selection in such an environment, a detector with a space resolution of the order 
of $1 \mathrm{~cm}$ and a time resolution better than $3 \mathrm{~ns}$ is required. Single gas gap Resistive Plate Chambers (RPCs) fulfill these requirements[10].

The trigger system is based on two large area $\left(5.5 \times 6.5 \mathrm{~m}^{2}\right)$ detector stations; each station consists of two planes of 18 RPCs each, equipped with orthogonal strips arranged in projective geometry relative to the interaction point. The strips are equipped with front-end electronics based on a dual threshold discriminator (ADULT[11]) which allows achieving a time resolution better than $2 \mathrm{~ns}$ in streamer mode. From the front-end, the signals are sent to the trigger electronics, based on programmable circuits.

The trigger algorithm makes use of the x-y information of the four RPC planes for reconstructing tracklets and performing the transverse momentum cut. Two different $p_{t}$ can be applied: $p_{t}>1 \mathrm{GeV} / \mathrm{c}$ and $p_{t}>2 \mathrm{GeV} / \mathrm{c}$. The system delivers, in 800ns, six different trigger signals: at least one muon track above low - (high-) $p_{t}$ cut, at least two unlike-sign muon tracks, each above low - (high-) $p_{t}$ cut, at least two like-sign muon track, each above low - (high-) $p_{t}$ cut.

Due to the specific requirements for the different colliding systems, the RPCs will be operated in streamer mode in ion-ion collisions where the expected occupancy is high, and in highly saturated avalanche mode in p-p collisions where the detector aging might be more relevant in view of the expected counting rate on long data taking periods. The same front-end electronics can be used for the two operation modes setting the discriminator thresholds at different values.

\section{Commissioning results}

All the detectors are now installed. The commissioning in the cavern started at the beginning of 2007 with the check of all the services (gas, $\mathrm{HV}, \mathrm{LV}$, cooling) and the optimization of several parameters: high voltage, electronic noise and pedestals, signals timing and trigger alignment at the level of the ALICE central trigger processor (CTP). Tests of the whole spectrometer have been performed with cosmic rays during two periods of data taking in 2009 where it was possible to understand the behavior and the integration of each detector element during the data taking. Efficiency measurements were carried out and the off-line reconstruction was tested with real tracks.

A remote control of the detector was developed in the Detector Control System (DCS) framework: on-line monitoring of the system is



Figure 2: One of the first muon pairs detected and reconstructed in the spectrometer therefore possible allowing to check stability in time.

During the first period the magnetic field was off and the spectrometer collected about 100k tracks while, in the last one, both configurations (magnet on/off) were used and about 470k tracks were collected. At the end of 2009 the muon spectrometer was fully operational for the first $p-p$ 
collisions and collected about $1.5 \mathrm{~K}$ single muon tracks and few muon pairs, one of which is shown in Fig.2.

\section{Trigger System Commissioning}

During the 2009 data taking period both trigger stations were fully operative and readout. Due to its geometrical acceptance, the ALICE muon spectrometer is not designed to detect cosmic rays, nevertheless the system was able to deliver trigger signals with a rate of $0.2 \mathrm{~Hz}$ for nearly horizontal tracks. In the first commissioning period with cosmic rays the RPCs have been operated in streamer mode, while during the second one in avalanche mode.

The RPC efficiency was studied by using the detection plane redundancy. Indeed, the trigger algorithm requires only three over four detection planes to be fired, allowing to study the fourth plane. The results show that the efficiency is larger than $90 \%$ for all RPCs both in streamer and in avalanche mode.

Varying the high voltage of a plane of RPCs, an efficiency scan was performed for all the planes in order to optimize the operation voltage of each RPC (an example is shown in Fig.3).

The counting rate (due to noise) and the current were measured for each RPCs. In streamer mode, where the RPC operation voltage is about $8 k V$, the average counting rate is relatively weak $\left(<R_{R P C}>=0.012 \mathrm{~Hz} / \mathrm{cm}^{2}\right)$ and the current distribution, which trend is stable in time, has a mean value of $0.4 \mu \mathrm{A}$. In avalanche mode, at a working

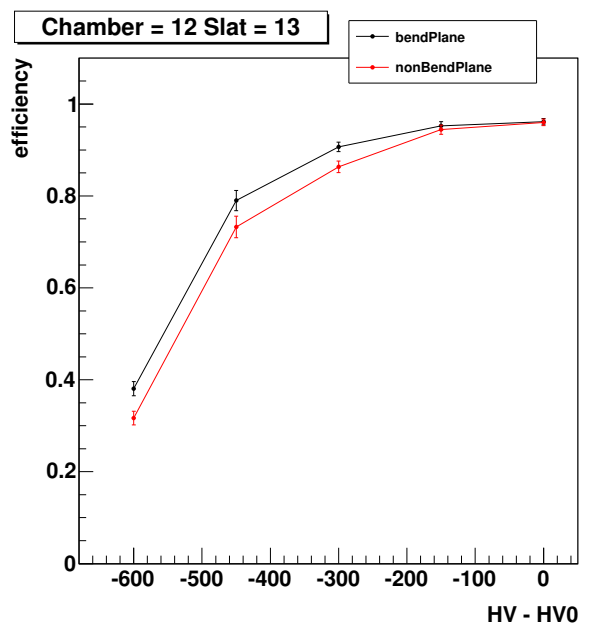

Figure 3: Efficiency scan of a RPC operating in streamer mode voltage of about $10.3 \mathrm{kV}$, these values are a little bit higher $\left(<R_{R P C}>=0.036 \mathrm{~Hz} / \mathrm{cm}^{2}\right.$ and $\left.\left\langle I_{R P C}\right\rangle=1.6 \mu \mathrm{A}\right)$ as expected from the higher working voltage and from the adopted thresholds. During p-p collisions, RPCs were operated in avalanche mode and the trigger electronics was successfully delivering trigger signals: a single muon trigger rate of $0.5 \%$ per p-p minimum-bias collision was measured.

\section{Tracking System Commissioning}

Starting from the last period of cosmic rays data taking the tracking system is in its final shape. All CPCs were powered at $1.6 \mathrm{kV}$. The noise level is below project requirements. The number of dead channels is modest and it doesn't affect the reconstruction efficiency due to the redundancy of the system: six hits out of the 10 detection planes are the minimum requirement for the track reconstruction, with at least one hit per station in the first 3 ones and three over four hits in the last two stations.

The cosmic ray data taking was very useful to test the off-line realignment. Millepede algorithm[12] was used. With 10k cosmic tracks passing through all the spectrometer (with no B field), an accuracy of the order of $500 \mu \mathrm{m}$ was reached for the bending plane coordinate. To reach values below 
$100 \mu \mathrm{m}$, as requested for the mass resolution of the $\Upsilon, 100 k$ tracks are needed and will be collected in p-p collisions.

\section{Conclusions}

After several years of R\&D and installation, the ALICE muon spectrometer is now fully operational. Most of its features are under control and its performance was demonstrated with the reconstruction of the first muon tracks from p-p collisions at $900 \mathrm{GeV}$.

The spectrometer is now ready for data tacking both in $\mathrm{p}-\mathrm{p}$ and in $\mathrm{Pb}-\mathrm{Pb}$ collisions.

\section{References}

[1] ALICE collaboration, The ALICE experiment at the CERN LHC, JINST 3 (2009) S08002.

[2] M.C. Abreu et al, Evidence for deconfinement of quarks and gluons from the J/ $\psi$ suppression pattern measured in Pb-Pb collisions at the CERN-SPS, Phys. Lett. B 477 28-36

[3] A. Adare et al. PHENIX Collaboration, $J / \psi$ Production versus Centrality, Transverse Momentum, and Rapidity in Au+Au Collisions at $\sqrt{s_{N N}}=200 \mathrm{GeV}$, Phys. Rev. Lett. 98, 232301 (2007)

[4] M. Bedjidian et al., Hard probes in heavy ion collisions at the LHC: heavy flavour physics, CERN Yellow Report CERN-2004-009 arXiv: hep-ph/0311048

[5] R. Baier et al., Energy Loss in Perturbative QCD, Ann. Rev. Nucl. Part. Sci. 50, (2000) 37.

[6] ALICE collaboration, ALICE: Physics Performance Report, Volume I, J. Phys. G: Nucl. Part. Phys. 32 (2006) 1295-2040.

[7] J. Peyré, B. Genolini and J. Pouthas, A full-scale prototype for the tracking chambers of the ALICE mиоn spectrometer, 1998 ALICE-INT 1998-28.

[8] Charpy A et al, Test of the tracking readout electronics of the Dimuon Forward Spectrometer, 2006 ALICE-INT 2006-016.

[9] R. Tieulent, The Geometry Monitoring System of the ALICE Dimuon Spectrometer - Overview, 2005 ALICE-INT 2005-009.

[10] R. Arnaldi et al., A low-resistivity RPC for the ALICE dimuon arm, Nucl. Instr. and Meth. A 451 (2000) 462.

[11] R. Arnaldi et al., A dual threshold technique to improve the time resolution of resistive plate chambers in streamer mode, Nucl. Instr. and Meth. A 457 (2001) 117.

[12] V. Blobel and C. Kleinwort, A New Method for the High-Precision Alignment of Track Detectors, Report DESY 02-077 (June 2002) [hep-ex/ 0208021 ] 